\title{
Primary HIV-I Infection Sets the Stage for Important B Lymphocyte Dysfunctions
}

Kehmia Titanji* ${ }^{*}$, Francesca Chiodi ${ }^{1}$, Rino Bellocco ${ }^{2}$, Danika Schepis ${ }^{1}$, Alberto Cagigi ${ }^{1}$, Lyda Osorio ${ }^{1}$, Chiara Tassandin ${ }^{3}$, Giuseppe Tambussi ${ }^{3}$, Sven Grutzmeier ${ }^{1}$, Lucia Lopalco ${ }^{3}$ and Angelo De Milito ${ }^{1}$

\author{
Address: ${ }^{1}$ Microbiology and Tumorbiology Center, Karolinska Institutet, Stockholm, Sweden, ${ }^{2}$ Department of Medical Epidemiology and \\ Biostatistics, Karolinska Institutet, Stockholm, Sweden and ${ }^{3}$ Infectious Disease Clinic, San Raffaele Scientific Institute, Milan, Italy \\ Email: Kehmia Titanji* - Kehmia.Titanji@mtc.ki.se \\ * Corresponding author $\ddagger$ Presenting author
}

from 2005 International Meeting of The Institute of Human Virology

Baltimore, USA, 29 August - 2 September 2005

Published: 8 December 2005

Retrovirology 2005, 2(Suppl I):P95 doi:10.1 186/I742-4690-2-SI-P95

\section{Background}

B lymphocytes of patients with chronic HIV-1 infection (CHI) show functional and phenotypic abnormalities. We investigated the effects of primary HIV-1 infection (PHI) on activation, differentiation and survival of $\mathrm{B}$ cells. The effects of antiretroviral therapies on B cell dysfunctions in PHI were also studied.

\section{Design and Methods}

B cells of 31 PHI patients (sampled at baseline, 1 month and 6 months post therapy), $26 \mathrm{CHI}$ patients, and 12 healthy donors were studied for surface expression of Fas, LAIR-1, CD70, intracellular expression of Bcl-2, and spontaneous apoptosis. Four-colour FACS (IgD+IgM+CD19+CD27), and short-term PBMC cultures to analyse induction of CD25 on B cells were performed in 5 PHI patients.

\section{Results}

In PHI, naïve and memory B lymphocytes were highly activated, manifested by hypergammaglobulinemia, altered expression of Fas and LAIR-1, and increased spontaneous apoptosis. Antiretroviral treatment improved the activation/differentiation status of B cells, reduced apoptosis to levels comparable to healthy individuals and restored the ability of $\mathrm{B}$ cells to respond to T-cell dependent activation. B cells of PHI patients on HAART recovered better compared to patients on RTI only. Data obtained on 5 PHI patients at baseline showed decreased IgM+ memory B cells and lower induction of CD25 expression on B cells upon T cell activation. These parameters were normalized after 6 months of antiretroviral treatment.

\section{Conclusion}

B cell dysfunctions in HIV-1 infection appear during primary infection and initiation of antiretroviral therapy early during infection may help preserve the $\mathrm{B}$ cell compartment. 